\title{
On Certain Finite Difference Schemes For Hyperbolic Systems
}

\author{
By John Gary
}

1. Introduction. The purpose of this paper is to describe a few finite difference schemes for the numerical solution of hyperbolic systems of partial differential equations. Our main concern will be the stability conditions for these schemes although some of these schemes may be useful, especially for problems involving supersonic fluid flow. We will describe numerical experiments designed to check the derived stability conditions and also the accuracy of these schemes.

In Section 2 we will describe the application of the Crank-Nicholson scheme to hyperbolic systems. This method is unconditionally stable, however it requires the inversion of a block tridiagonal matrix. We describe two modifications which require only the inversion of a scalar tridiagonal matrix. We carry out a stability analysis which shows that these schemes are unconditionally stable only if the matrix of the system is positive definite (supersonic flow in the case of hydrodynamics). Results of numerical computations using all these schemes are described in Section 3. All our results are for problems in one space dimension. These methods can be generalized to two dimensions but we have no analysis to indicate that the generalizations will work. In Section 5 we give the results of fluid dynamics computations using three versions of the Lax-Wendroff difference scheme. The objective here is to determine if it is necessary to write the equations in conservation form in order to obtain good results when the flow contains a shock. In Section 6 an application of the Lax-Wendroff scheme to the Navier-Stokes equations is described. An empirical stability condition is obtained which is a combination of the usual hyperbolic and parabolic conditions. In Section 7 an explicit difference scheme related to the CrankNicholson scheme is given. This is an iterative scheme with a rather peculiar stability condition.

The author is indebted to several staff members at the Courant Institute of Mathematical Sciences for advice and encouragement, especially Professors R. D. Richtmyer and H. B. Keller. The computations described herein were all carried out on the IBM 7090 at New York University.

2. Description of the Finite Difference Schemes. In this section we will describe some finite difference schemes of an implicit nature. For purposes of comparison we will begin with the usual Crank-Nicholson scheme [4]. This scheme is adapted to the nonlinear problem by use of a "predictor-corrector" method. We denote the hyperbolic system of equations by $\partial w / \partial t+A(w) \partial w / \partial x=0$ where $A(w)$ is a matrix with real, distinct eigenvalues. If the equations are those of hydrodynamic flow for a polytropic gas we have

$$
A(w)=\left[\begin{array}{ccc}
u & 0 & \rho \\
0 & u & \gamma p \\
0 & 1 / \rho & u
\end{array}\right], \quad w=\left[\begin{array}{c}
\rho \\
p \\
u
\end{array}\right],
$$

Received July 31,1962 . Revised July 10, 1963. This research was carried out under contract with the U. S. Atomic Energy Commission. 
where $\rho, p$, and $u$ denote density, pressure and velocity and $\gamma$ is the ratio of specific heats. We will use a mesh of equally spaced points $\left(x_{j}, t_{n}\right)$ where $x_{j+1}-x_{j}=$ $\Delta x, t_{n+1}-t_{n}=\Delta t$ and $1 \leqq j \leqq M$. We use the notation $w_{j}{ }^{n}=w\left(x_{j}, t_{n}\right)$,

$$
\begin{aligned}
& w_{x}=\frac{w(x+\Delta x, t)-w(x, t)}{\Delta x}, \\
& w_{\bar{x}}=\frac{w(x, t)-w(x-\Delta x, t)}{\Delta x}, \\
& w_{\hat{x}}=\frac{w(x+\Delta x, t)-w(x-\Delta x, t)}{2 \Delta x} .
\end{aligned}
$$

We shall assume the boundary values $w\left(x_{1}, t\right)$ and $w\left(x_{M}, t\right)$ are known and constant. This problem is probably not properly posed. If we were solving the equations $\partial w / \partial t+A \partial w / \partial t$ where $A$ is a constant matrix with distinct positive eigenvalues, then we should specify the initial values $w(x, 0)$ and the boundary values $w\left(x_{1}, t\right)$ but not $w\left(x_{M}, t\right)$ as an analysis of the direction of the characteristics will show. However, the specification of all dependent variables at both boundary points seemed to cause no trouble in our computations.

The first step in the Crank-Nicholson method is to predict the values of $w_{j}{ }^{n+1}$ by the use of an explicit difference scheme [10]. We denote the predicted values by $w^{+}$, and define them by

$$
w^{+}=w^{n}-\Delta t A\left(w^{n}\right) w_{\hat{x}}^{n} .
$$

Here we have suppressed the spatial index $j$. When written out in full these equations are

$$
w_{j}^{+}=w_{j}^{n}-\Delta t A\left(w_{j}^{n}\right)\left(w_{j+1}^{n}-w_{j-1}^{n}\right) / 2 \Delta x .
$$

Then $w^{n+1}$ is defined by the boundary conditions and the following equations

$$
w^{n+1}=w^{n}-\frac{\Delta t}{2} A\left(\frac{w^{n}+w^{+}}{2}\right)\left(w_{\hat{x}}^{n+1}+w_{\hat{x}}{ }^{n}\right) .
$$

The truncation error is $O\left(\Delta x^{2}+\Delta t^{2}\right)$. If the boundary values $w_{1}{ }^{n}$ and $w_{M}{ }^{n}$ are given then we require the above equations to hold for $j=2, \cdots, M-1$. When we perform a stability analysis on any of these schemes we will assume the boundary conditions are periodic, that is, $w_{j}{ }^{n}=w_{j+M}^{n}$. Then we assume the difference equations hold for $j=1,2, \cdots, M$.

To solve these equations we use a method given by S. Schechter [5]. We may write the system as

$$
\beta A_{j} w_{j+1}^{n+1}+w_{j}^{n+1}-\beta A_{j} w_{j-1}^{n+1}=D_{j},
$$

where $\beta=\Delta t /(4 \Delta x), A_{j}=A\left(\left(w_{j}{ }^{n}+w_{j}{ }^{+}\right) / 2\right)$ and $D$ is a vector defined by

$$
D_{j}=w_{j}^{n}-\beta A_{j}\left(w_{j+1}^{n}-w_{j-1}^{n}\right) .
$$

Thus the matrix of this system is block tridiagonal. The method is analogous to that used for a scalar tridiagonal system. We define matrices $F_{j}$ and vectors $G_{j}$ by recursion as follows: $F_{1}=I, G_{1}=D_{1}, F_{j}=I+\beta^{2} A_{j} F_{j-1}^{-1} A_{j-1}$, and $G_{j}=$ 
$D_{j}+\beta A_{j} F_{j-1}^{-1} D_{j-1}$. Then the solution is obtained by "backward substitution" $w_{M}{ }^{n+1}=F_{M}{ }^{-1} G_{M}, w_{j}{ }^{n+1}=F_{j}^{-1}\left(G_{j}-\beta A_{j} w_{j+1}^{n+1}\right)$.

To use this method we must be certain that the matrices $F_{j}$ can be inverted without difficulty. If the matrix $A(w)$ is constant, then $A$ has a complete set of distinct eigenvectors. These are also a complete set for the matrices $F_{j}$. If $\alpha$ is an eigenvalue of $A_{j}$ and $\lambda_{j}$ is the corresponding eigenvalue of $F_{j}$, then $1+\beta^{2} \alpha^{2} / \lambda_{j}$ is the corresponding eigenvalue of $F_{j+1}$. Therefore $1 \leqq\left\|F_{j}\right\| \leqq 1+a^{2}$ for $1 \leqq j \leqq M$, where $a \geqq \beta\|A\|$. If the matrices $A$ are all symmetric, then the norm of $F_{j}$ satisfies the same inequality. In our case the matrices $A_{j}$ are determined by equations (2.1) and are therefore neither symmetric nor constant. However, we had no difficulty in inverting the matrices $F_{j}$. An analysis by the method of von Neumann [4] shows this scheme to be unconditionally stable and this conclusion is supported by numerical experiments described in the following section.

We will next define a quasi-Crank-Nicholson $(Q-C-N)$ scheme which is not centered in time. We define the lower triangular matrix $A_{L}$ to consist of those elements of $A$ on and below the diagonal with zero elements above the diagonal. We define $A_{U}$ by $A_{U}=A-A_{L}$. Then the (Q-C-N) difference scheme is defined by

$$
w^{n+1}=w^{n}-\Delta t A_{L}\left(w^{n}\right) w_{\hat{x}}^{n+1}-\Delta t A_{U}\left(w^{n}\right) w_{\hat{x}}^{n} .
$$

To solve for each dependent variable we need only solve a scalar tridiagonal system of equations. Thus the scheme is effectively explicit. This is the reason for splitting the matrix into triangular parts. If the matrix $A(w)$ is defined by equations $(2.1)$, then this scheme is unconditionally stable for supersonic flow and unconditionally unstable for subsonic flow. This will be discussed in Section 4. The truncation error is $O\left(\Delta t+\Delta x^{2}\right)$.

We can also define a quasi-Crank-Nicholson scheme which is centered in time. The first step is to predict the values of $w^{n+1}$ by the equations

$$
w^{+}=w^{n}-\Delta t A\left(w^{n}\right) w_{\hat{x}}^{n} .
$$

The centered $\mathrm{Q}-\mathrm{C}-\mathrm{N}$ scheme is then defined by the equations

$$
w^{n+1}=w^{n}-\frac{\Delta t}{2} A_{L}{ }^{+} w_{\hat{x}}^{n+1}-\frac{\Delta t}{2} A_{U}{ }^{+} w_{\hat{x}}{ }^{+}-\frac{\Delta t}{2} A^{+} w_{\hat{x}}{ }^{n},
$$

where $A^{+}=A\left(\left(w^{n}+w^{+}\right) / 2\right)$. The solution of this system is obtained by solving a tridiagonal matrix equation for each dependent variable. The truncation error is $O\left(\Delta t^{2}+\Delta x^{2}\right)$. In Section 4 we will show that this scheme is unconditionally stable for supersonic flow and conditionally stable for subsonic flow, provided that the matrix $A$ is given by equations (2.1).

This centered quasi-Crank-Nicholson scheme was applied to the Navier-Stokes equations:

$$
\begin{gathered}
\frac{\partial \rho}{\partial t}+u \frac{\partial \rho}{\partial x}+\rho \frac{\partial u}{\partial x}=0 \\
\frac{\partial T}{\partial t}+u \frac{\partial T}{\partial x}+(\gamma-1) T \frac{\partial u}{\partial x}=\frac{\gamma}{P_{r} R \rho} \frac{\partial^{2} T}{\partial x^{2}}+\frac{4 \gamma}{3 \rho R}(\gamma-1)\left(\frac{\partial u}{\partial x}\right)^{2} \\
\frac{\partial u}{\partial t}+u \frac{\partial u}{\partial x}+\frac{1}{\gamma} \frac{\partial T}{\partial x}+\frac{T}{\gamma \rho} \frac{\partial \rho}{\partial x}=\frac{4}{3 \rho R} \frac{\partial^{2} u}{\partial x^{2}} .
\end{gathered}
$$


In these equations $R$ represents the Reynolds number, $P_{r}$ the Prandtl number, and $T$ the temperature. In Section 6 we find that the Lax-Wendroff method applied to the Navier-Stokes equations yields the following approximate stability relation

$$
\Delta t<\min \left[\frac{\Delta x}{|u|+c}, \frac{3 R \rho \Delta x^{2}}{16}\right] .
$$

There is a parabolic dependence on $\Delta x$. We have not determined a stability criterion for the centered Q-C-N method applied to the Navier-Stokes equations. However, enough computations were carried out to show that the stability condition depends on $\Delta x^{2}$ very weakly, if at all. Thus the stability condition is less stringent, especially for values of $R$ which are not large.

It might be possible to use these methods for problems in two space dimensions by using alternating direction techniques. For example, suppose we wish to solve the system

$$
\frac{\partial w}{\partial t}+A(w) \frac{\partial w}{\partial x}+B(w) \frac{\partial w}{\partial y}=0 .
$$

The finite difference scheme could be

$$
\begin{aligned}
w^{+} & =w^{n}-\Delta t A_{L}{ }^{0} w_{\hat{x}}^{+}-\Delta t A_{U}{ }^{0} w_{\hat{x}}{ }^{n}-\Delta t B^{0} w_{\hat{y}}{ }^{n}, \\
w^{n+1} & =w^{n}-\Delta t A^{1} w_{\hat{x}}{ }^{+}-\Delta t B_{L}{ }^{1} w_{\hat{y}}{ }^{n+1}-\Delta t B_{U}{ }^{1} w_{\hat{y}}{ }^{+},
\end{aligned}
$$

where $A^{0}=A\left(w^{n}\right)$ and $A^{1}=A\left(\left(w^{+}+w^{n}\right) / 2\right)$. If this scheme is stable at all, it would probably be stable only for supersonic flow. The fact that the truncation error is first order in $\Delta t$ may not be a major defect, particularly if the method is used to solve for a steady state solution. Perhaps it is possible to devise a scheme which is unconditionally stable for both subsonic and supersonic flow which involves nothing worse than the solution of scalar tridiagonal matrices.

3. Results of Numerical Computations. To test these schemes we used a hydrodynamic flow containing a simple rarefaction or compression wave. We applied the difference schemes to equations (2.1.). The initial function $u(x, 0)$ was continuous, constant around $x_{1}$ and $x_{M}$ and a monotone polynomial in the interval in which it was not constant. For example:

$$
u(x, 0)= \begin{cases}u_{0} & x_{1} \leqq x \leqq a \\ q(x)= & u_{0}+\frac{x-a}{b-a}\left(u_{1}-u_{0}\right) \\ u_{1} & b \leqq x \leqq x_{M} .\end{cases}
$$

By varying $q(x)$ it was possible to have $u(x, 0) \in C^{0}, C^{1}, C^{2}$, or $C^{3}\left(u \in C^{3}\right.$ means $u$ has a continuous third derivative). The initial values $p(x, 0)$ and $p(x, 0)$ were computed from the equations below. This produces a simple wave moving on the characteristic with slope $u+c$. In our formulation we could not use the scheme after the wave reached the downstream boundary since we assumed $u\left(u_{M}, t\right)$ was constant.

$$
\rho(x, 0)=\rho_{0}\left[1+\frac{\gamma-1}{2} \frac{\left(u-u_{0}\right)}{c_{0}}\right]^{2 /(\gamma-1)} \quad p(x, 0)=\frac{p_{0}}{\rho_{0}} \rho(x, 0)^{\gamma} .
$$


TABLE I

Percentage error after 100 time steps for the various difference schemes

\begin{tabular}{|c|c|c|c|c|c|c|}
\hline $\begin{array}{l}\text { No. of } \\
\text { Points }\end{array}$ & $\begin{array}{c}\mathrm{C}-\mathrm{N} \\
(\text { A cen.) }\end{array}$ & $\begin{array}{c}\mathrm{C}-\mathrm{N} \\
(\mathrm{A} \text { not cen.) }\end{array}$ & $\begin{array}{c}\text { Q-C-N } \\
\text { (cen.) }\end{array}$ & $\begin{array}{l}\text { Q-C-N } \\
(\text { cen.) } \\
u \in \text { C }^{3}\end{array}$ & $\begin{array}{c}\mathrm{Q}-\mathrm{C}-\mathrm{N} \\
\text { (not cen.) }\end{array}$ & $\begin{array}{l}\text { Lax- } \\
\text { Wend. }\end{array}$ \\
\hline $\begin{array}{r}10 \\
20 \\
100 \\
200\end{array}$ & $\begin{array}{l}4.4 \% \\
1.7 \\
0.055 \\
0.0087\end{array}$ & $\begin{array}{l}4.7 \% \\
1.9 \\
0.11 \\
0.042\end{array}$ & $\begin{array}{l}4.1 \% \\
1.6 \\
0.047 \\
0.0073\end{array}$ & $\begin{array}{l}4.8 \% \\
1.8 \\
0.039 \\
0.0050\end{array}$ & $\begin{array}{l}8.8 \% \\
5.2 \\
0.57 \\
0.15\end{array}$ & $\begin{array}{l}2.0 \% \\
0.71 \\
0.014 \\
0.0020\end{array}$ \\
\hline $\begin{array}{l}\text { Computation time } \\
\text { time in milli- } \\
\text { seconds per } \\
\text { mesh point }\end{array}$ & 15 & 13 & 4.4 & 4.4 & 1.7 & 3.8 \\
\hline
\end{tabular}

In these equations $\rho_{0}, p_{0}, c_{0}$ represent the constant state ahead of the wave. The exact solution to this problem is easily calculated [1].

The results of the computation are shown in Table I. The number of points in the first column refers to the number of mesh points used to cover the interval over which $u(x, 0)$ is not constant. This determined the value of $\Delta x$. The value of $\Delta t$ is given by $\Delta t=0.9 \Delta x /(|u|+c)$ which is the Courant-Friedrichs-Lewy condition for stability. The uncentered Crank-Nicholson (C-N) scheme refers to one in which the coefficient matrix is not centered, that is:

$$
w^{n+1}=w^{n}-\Delta t A\left(w^{n}\right)\left(\frac{w_{\hat{x}}^{n+1}+w_{\hat{x}}^{n}}{2}\right) .
$$

In one case the quasi-Crank-Nicholson scheme was run with an initial value $u(x, 0)$ which had a continuous third derivative. In the other cases $u(x, 0) \in C^{2}$ but $\partial^{3} u / \partial x^{3}$ was discontinuous at the head and tail of the rarefaction wave. If the truncation error is $O\left(\Delta x^{2}\right)$, the percentage error should drop by a factor of eight when the mesh spacing is halved. The percentage error given in the table is the maximum percentage error in the variables $\rho, p, u$ throughout the mesh at 100 time steps. If the truncation error is to be $O\left(\Delta x^{2}\right)$, we must have $u \in C^{3}$. The flow is a simple rarefaction wave with Mach number $M_{0}=2$ ahead of the wave and $M_{1}=1.75$ behind the wave. Other runs were made with $M_{0}=0$ and $M_{1}=0.7$. In this case the error was less than that shown in the table. The computing time shown at the bottom of the table is the time required (in milli-seconds) to compute the solution at a single mesh point. Results of computing with the Lax-Wendroff scheme are shown for comparison. The equations used in the Lax-Wendroff scheme are written in quasiconservation form (see Section 5). In Table I, "C-N" denotes Crank-Nicholson, "Q-C-N" denotes quasi-Crank-Nicholson and "cen." denotes a centered scheme. Obviously the Lax-Wendroff scheme is superior in accuracy and computation time.

4. Derivation of Stability Conditions. We will first derive a stability condition for the centered quasi-Crank-Nicholson scheme with the matrix $A(w)$ given by equations (2.1). We use the method of von Neumann, that is, we linearize the equations and assume a perturbation of the form $w^{n}=w\left(x_{j}, t_{n}\right)=k^{n} \exp \left(i \omega x_{j}\right)$. 
Substituting this value for $w_{j}{ }^{n}$ into equations (2.3) and (2.4) we obtain

$$
\begin{aligned}
k^{n+1} & =\left\{I-\left[I+\frac{i \beta}{2} A_{L}\right]^{-1}\left[I-\frac{i \beta}{2} A_{U}\right] i \beta A\right\} k^{n} \\
& =R k^{n},
\end{aligned}
$$

where $\beta=(\Delta t \sin \omega \Delta x) / \Delta x$. If we let $\alpha=\beta u / 2$, then the amplification matrix $R$ is given by $R=I+2 \alpha F /(1+i \alpha)$ where the matrix $F$ is given by

$$
F=\left[\begin{array}{ccc}
-i & -\frac{\alpha}{u^{2}} & -\frac{i \rho(1-i \alpha)}{u} \\
0 & -\left(i+\frac{\alpha}{M^{2}}\right) & -\frac{i \rho u(1-i \alpha)}{M^{2}} \\
0 & \frac{i\left(\alpha^{2} / M^{2}-1\right)}{\rho u(1+i \alpha)} & -\frac{\alpha}{M^{2}} \frac{(1-i \alpha)}{(1+i \alpha)}-i
\end{array}\right] .
$$

Here $M$ denotes the Mach number. If we denote the eigenvalues of $F$ by $\mu_{j}(j=$ $1,2,3)$, then the eigenvalues of $R$ are $\left(1+i \alpha+2 \alpha \mu_{j}\right) /(1+i \alpha)$. Let $z_{j}=1+$ $i \alpha+2 \alpha \mu_{j}$; then by expanding the characteristic equation of $F$ to find an equation for $\mu_{j}$ we obtain $z_{3}=1-i \alpha,\left(z_{j}+Q_{1}\right)\left(z_{j}+Q_{2}\right)=-Q_{3}(j=1,2)$ where

$$
\begin{aligned}
& Q_{1}=\alpha i+\frac{2 \alpha^{2}}{M}-1, \\
& Q_{2}=\alpha i+\frac{2 \alpha^{2}(1-i \alpha)}{M^{2}(1+i \alpha)}-1, \\
& Q_{3}=\frac{4 \alpha^{2}\left(1-\alpha^{2} / M^{2}\right)(1-i \alpha)}{M^{2}(1+i \alpha)} .
\end{aligned}
$$

Then $z_{1}+z_{2}=-\left(Q_{1}+Q_{2}\right)=(1-i \alpha)\left(2-4 \alpha^{2} /\left(M^{2}\left(1+\alpha^{2}\right)\right)\right.$ and $z_{1} z_{2}=(1-$ $i \alpha)^{2}$. Let $z_{j}=a_{j}(1-i \alpha)$, then $a_{1} a_{2}=1$ and $a_{1}+a_{2}=2-4 \alpha^{2} /\left(M^{2}\left(1+\alpha^{2}\right)\right)$. The eigenvalues of $R$ are $r_{3}=(1-i \alpha) /(1+i \alpha)$ and $r_{j}=z_{j} /(1+i \alpha)(j=1,2)$. Thus $\left|r_{3}\right|=1$, and $\left|r_{j}\right|=\left|a_{j}\right|(j=1,2)$. Therefore $\left|r_{j}\right| \leqq 1(j=1,2,3)$ if and only if $\alpha^{2} \leqq M^{2}\left(1+\alpha^{2}\right)$. This follows from the product and sum relations for $a_{j}$. Therefore we have unconditional stability if $M \geqq 1$. If $M<1$, then the inequality $\alpha^{2} \leqq M^{2}\left(1+\alpha^{2}\right)$ reduces to $\beta^{2} c^{2} \leqq 4+\beta^{2} u^{2}$ or $\Delta t \leqq 2 \Delta x / \sqrt{c^{2}-u^{2}}$ where $c$ is the velocity of sound. This completes the stability analysis.

We will now analyze the stability of the uncentered scheme defined by equations (2.2). Again we use the von Neumann method. The amplification matrix $R$ is $R=C_{1}^{-1} C_{2}$ where $C_{1}=I+\beta A_{L}, C_{2}=I-\beta A_{U}$, and $\beta=(i \Delta t \sin \omega \Delta x / \Delta x$. We first assume that $A(w)$ is constant, symmetric and positive definite. We can then prove that $\sigma(R) \leqq 1$. The proof is almost exactly the same as that given by Ostrowski to prove that the Gauss-Seidel iterations converge [2]. Assume, for simplicity, that the order of $A$ is three. Let $e_{1}=(1,0,0), e_{2} \stackrel{=}{=}(0,1,0)$ and $e_{3}=(0$, $0,1)$. Let $x_{0}$ be an arbitrary three-dimensional vector, and let $[x]_{i}$ denote the $i$-th component of a vector $x$. Let $x_{1}=x_{0}+\alpha_{1} e_{1}, x_{2}=x_{1}+\alpha_{2} e_{2}$ and $x_{3}=x_{2}+\alpha_{3} e_{3}$ where $\alpha_{i}$ is chosen such that $\left[C_{1} x_{j}\right]_{j}=\left[C_{2} x_{j-1}\right]_{j}(j=1,2,3)$. It is easy to show that $C_{1} x_{3}=C_{2} x_{0}$. We continue this process to obtain a sequence of vectors $x_{j}$ such that 
$C_{1} x_{3 j}=C_{2} x_{3 j-3}$. We will show that $\left|x_{j}\right| \rightarrow 0$ (for $\beta \neq 0$ ) which in turn implies $\lim R^{n} x_{o}=0$. Thus we will have proved stability. Since $A$ is symmetric and positive definite we can define a norm for complex vextors $x$ by $\|x\|=x^{*} A x$. We have $x_{j+1}=x_{j}+\alpha_{j+1} e_{k_{j}}$ where $1 \leqq k_{j} \leqq 3$. Hereafter we omit the subscript in $k_{j}$. A little algebra will yield

$$
\left\|x_{j}\right\|-\left\|x_{j-1}\right\|=2 \operatorname{Re}\left\{\bar{\alpha}_{j}\left[A x_{j-1}\right]_{k}\right\}+\bar{\alpha}_{j} \alpha_{j} a_{k k} .
$$

From the definition of $x_{j}$ we have $\left[C_{1} x_{j}-C_{2} x_{j-1}\right]_{k}=0$. From this we have $\left[\beta A x_{j}\right]_{k}=$ $\alpha_{j}\left(1-\beta a_{k k}\right)$. Therefore $\left\|x_{j+1}\right\|-\left\|x_{j}\right\|=-\alpha_{j} \bar{\alpha}_{j} a_{k k}<0$. This in turn yields the convergence of $\left\|x_{j+1}\right\|$ which shows that $\alpha_{j} \rightarrow 0$. Since $\left[\beta A x_{j}\right]_{k}=\alpha_{j}\left(1-\beta a_{k k}\right)$ we can prove that $x_{j} \rightarrow 0$.

If the matrix $A$ is given by equations (2.1) we are no longer able to obtain a stability condition by analytic means. However we can compute the eigenvalues of the amplification matrix numerically and thus determine a stability condition. The difference equation is

$$
w^{n+1}=w^{n}-\Delta t A_{L}\left(w^{n}\right) w_{\hat{x}}^{n+1}-\Delta t A_{U}\left(w^{n}\right) w_{\hat{x}}^{n} .
$$

If $A(w)$ is defined by equations (2.1), then the amplification matrix for this scheme is

$$
R=\left[\begin{array}{ccc}
\frac{1}{1+i \alpha} & 0 & -\frac{i \beta \rho}{1+i \alpha} \\
0 & \frac{1}{1+i \alpha} & -\frac{i \beta \gamma p}{1+i \alpha} \\
0 & \frac{-i \beta}{\rho(1+i \alpha)^{2}} & \frac{-\beta^{2} \gamma p}{\rho(1+i \alpha)^{2}}+\frac{1}{1+i \alpha}
\end{array}\right]
$$

where $\beta=(\Delta t \sin \omega \Delta x) / \Delta x$ and $\alpha=\beta u$. The eigenvalues of $R$ are given by $\mu_{j} /$ $(1+i \alpha)$ where $\mu_{3}=1$ and $\mu_{j}(j=1,2)$ are the roots of the following quadratic

$$
\mu^{2}-(2-\delta) \mu+1=0, \quad \delta=\frac{\beta^{2} c^{2}}{1+i \alpha}=\frac{\sigma^{2}}{1+i \sigma M} .
$$

Note that the eigenvalues of $R$ depend only on $\sigma=\beta c$ and $M=u / c$. We let $\lambda$ denote the absolute value of the largest eigenvalue of $R$. In Figure $1, \lambda$ is plotted against $\sigma$ for various values of the Mach number $M$. These graphs indicate that the scheme is unconditionally stable for $M>1$ and unconditionally unstable for $M<1$. Numerical computations were performed using this scheme as described in Section 3. The flow was a rarefaction wave with $M=2.0$ ahead and $M=1.75$ behind the wave. If the value of $\Delta t$ was made equal to ten times the value of Courant-Friedrichs-Lewy (i.e., $\Delta t=10 \Delta x /(|u|+c)$ ), then there was no sign of instability out to 44 time steps. Computations were also performed with a subsonic rarefaction wave with $M=0.0$ ahead and $M=0.7$ behind the wave. With $\Delta t=$ $2 \Delta x /(|u|+c)$ the density became negative at 61 time steps and when $\Delta t=$ $\Delta x /(|u|+c)$ the density was negative at 377 time steps. For small values of $\Delta t$ the largest eigenvalue of the amplification matrix $R$ is very close to the unit circle, therefore instability is slow to develop.

The eigenvalues of $M$ drop off quite rapidly for supersonic flow according to 


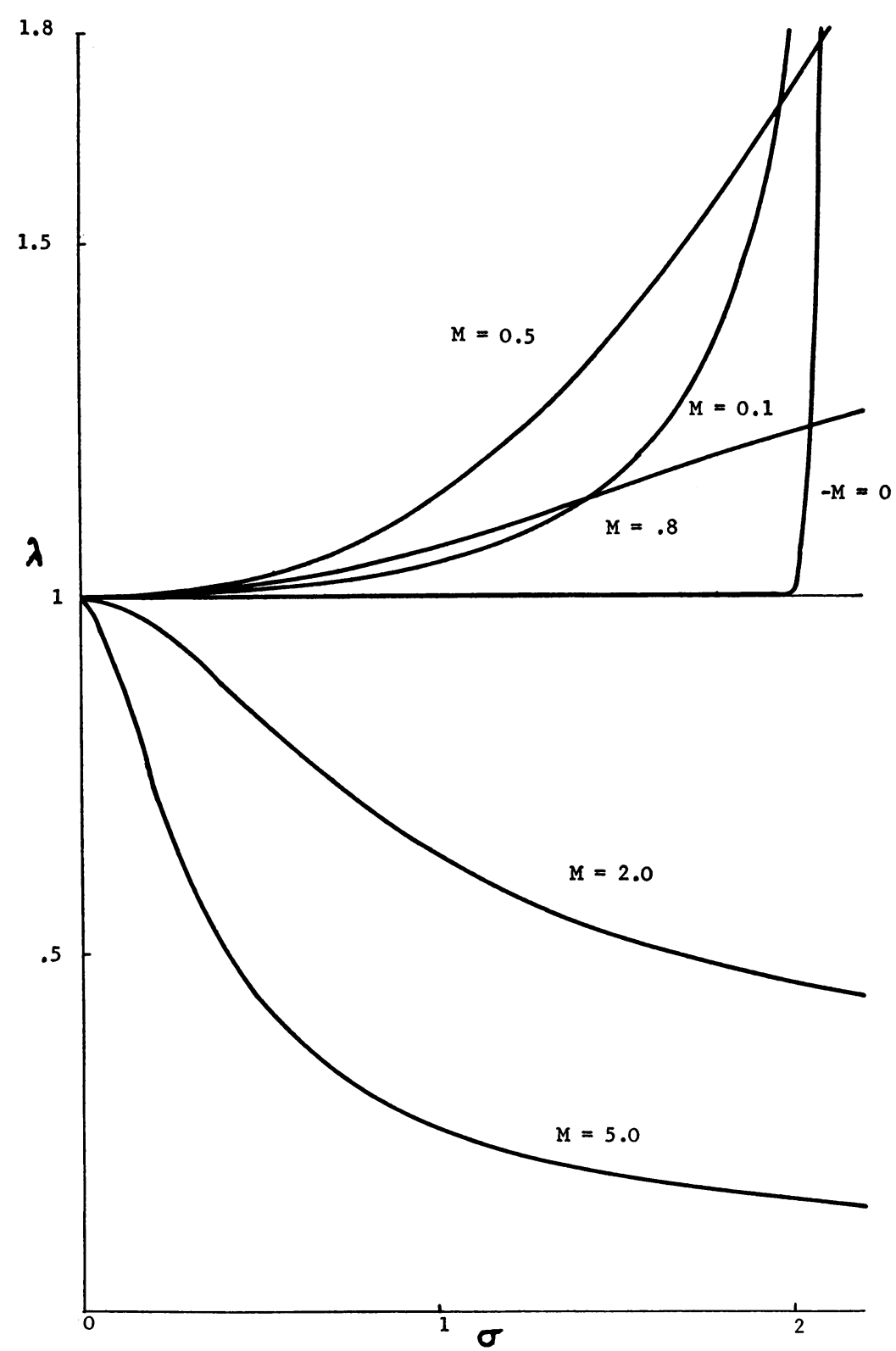

Fig. 1. Wave parameter $\sigma$ v.s. spectral radius $\lambda$ for $\mathrm{Q}-\mathrm{C}-\mathrm{N}$ scheme (uncentered).

Figure 1. This suggests that the scheme might produce reasonable results even $i$ the flow contains a shock. We tested this scheme on a flow which was initially an isentropic compression wave. Eventually the compression wave will produce a shock. This provides a test of how the difference scheme will react to the shock. We ran the Lax-Wendroff scheme on the same flow for a comparison. The initial compression wave had a Mach number of 2.53 behind and 1.5 ahead, which produced a very strong shock. The computed values of pressure are plotted in Figure 2. Both 
TABLE II

Consistency check from the Hugoniot relations

\begin{tabular}{|c|c|c|c|c|}
\hline & \multicolumn{2}{|c|}{ Lax-Wendroff } & \multicolumn{2}{|c|}{ Quasi-C-N } \\
\hline & Observed & $\begin{array}{l}\text { Computed } \\
\text { from R-H }\end{array}$ & Observed & $\begin{array}{l}\text { Computed } \\
\text { from R-H }\end{array}$ \\
\hline $\begin{array}{l}\text { Shock Mach number } \\
\text { Pressure ratio } \\
\text { Density ratio } \\
\text { Velocity behind }\end{array}$ & $\begin{array}{c}3.00 \\
10.4 \\
3.84 \\
3.70\end{array}$ & $\begin{array}{c}-\overline{10} \\
3.85 \\
3.72\end{array}$ & $\begin{array}{c}2.33 \\
10.7 \\
5.4 \\
3.65\end{array}$ & $\begin{array}{l}-\overline{17} \\
6.12 \\
3.11\end{array}$ \\
\hline
\end{tabular}

schemes were run to 300 time steps (at $\Delta x=0.05$ ). By printing out the computed value of pressure every 40 time steps from 100 to 300 time steps and observing where the shock is located we can compute the shock velocity. For both schemes the shock velocity is constant within the accuracy of measurement. We know the state ahead of the shock; thus we can compute the state behind the shock from the shock velocity and the Rankine-Hugoniot relations. The results are given in Table II.

When $\Delta x$ is reduced to 0.02 the results from the quasi-C-N scheme do not improve. The observed shock speed is 2.34 for $\Delta x=0.02$. This lack of improvement is to be expected since the state immediately behind the shock is nearly constant. Thus the results from the $\mathrm{Q}-\mathrm{C}-\mathrm{N}$ scheme are apparently worthless, although the curves in Figure 2 appear reasonable upon casual inspection.

5. The Lax-Wendroff Difference Scheme. In this section we shall be concerned with three versions of the Lax-Wendroff (L-W) difference scheme [6]. One may think of the L-W scheme as having an "artificial viscosity" built into it [7]. Therefore it works reasonably well on hydrodynamic flows which contain a shock discontinuity. Two versions of the $\mathrm{L}-\mathrm{W}$ scheme are based on the equations of hydrodynamics in conservation form in which the dependent variables are mass, momentum, and energy per unit volume, $(\rho, e, m)$ where $m=\rho u$ and $e=.5 \rho u^{2}+$ $p /(\gamma-1)$. The third version is based on the variables $(\rho, p, u)$. We will test these three versions to see how much improvement results from the use of conservation variables on flows which contain a shock.

The first version is exactly that proposed by Lax and Wendroff. We write the equations of flow in the form $\partial w / \partial t+\partial f / \partial x=0$ where $w=(\rho, e, m)$ and

$$
f(w)=\left(m, \frac{\gamma e m}{\rho}-\frac{\gamma-1}{2} \frac{m^{3}}{\rho^{2}},(\gamma-1) e-\frac{\gamma-3}{2} \frac{m^{3}}{\rho}\right) .
$$

We let $\lambda=\Delta t / \Delta x$ and

$$
q_{j}=\left(\frac{A\left(w_{j+1}^{n}\right)+A\left(w_{j}{ }^{n}\right)}{2}\right)\left(f\left(w_{j+1}^{n}\right)-f\left(w_{j}{ }^{n}\right)\right)
$$

where $A$ denotes the Jacobian of $f(w)$ with respect to $w$. Then the difference equations are

$$
w_{j}^{n+1}=w_{j}^{n}-\frac{\lambda}{2}\left(f\left(w_{j+1}^{n}\right)-f\left(w_{j-1}^{n}\right)\right)+\frac{\lambda^{2}}{2}\left(q_{j}-q_{j-1}\right) .
$$






Fig. 2. Pressure after 300 time steps $(\Delta x=.05)$.

This difference scheme is itself conservative in the sense that the following relations hold.

$$
\Delta x \sum_{j=1}^{M} w_{j}^{n+1}=\Delta x \sum_{j=1}^{M} w_{j}^{n}+\frac{\Delta t}{2}\left(f_{1}^{n}+f_{2}^{n}-f_{M-1}^{n}-f_{M-2}^{n}\right)+\frac{\Delta t^{2}}{2}\left(q_{M}-q_{1}\right) .
$$

These equations state that the sum of the mass, energy and momentum is the same at the two time levels except for the "flux" through the boundary. 
The second scheme is a slight modification of the first given below. It is not conservative in the above sense; we will say that it is in quasi-conservation form.

$$
w_{j}^{n+1}=w_{j}^{n}-\Delta t f_{\hat{x}}^{n}+\frac{\Delta t^{2}}{2}\left(A f_{\dot{x}}\right)_{x} .
$$

The third version uses the variables $(\rho, p, u)$. Like the first two versions it is based on a Taylor's series expansion of $u(x, t)$,

$$
u^{n+1}=u^{n}+\Delta t \frac{\partial u}{\partial t}+\frac{\Delta t^{2}}{2} \frac{\partial^{2} u}{\partial t^{2}}
$$

To convert this into a difference equation we need difference approximations for $\partial u / \partial t$ and $\partial^{2} u / \partial t^{2}$. The first we obtain from the flow equations, $\partial u / \partial t \sim-A w_{\hat{x}}$ where $A$ and $w$ are given by equations 2.1 . The second we obtain by differentiating the equations of motion. We then obtain three equations of the form

$$
\frac{\partial^{2} p}{\partial t^{2}}=-\gamma \frac{\partial p}{\partial t} \frac{\partial u}{\partial x}-\gamma p \frac{\partial^{2} u}{\partial t \partial x}-\frac{\partial u}{\partial t} \frac{\partial p}{\partial x}-u \frac{\partial^{2} p}{\partial t \partial x}
$$

Using the flow equations we obtain difference approximations for the first time derivatives in the form

$$
\delta p=-\gamma p u_{\bar{x}}-u p_{\dot{x}} .
$$

From the two preceding equations we obtain difference approximations to the second derivatives in the form

$$
\delta^{2} \rho=\delta \rho u_{x}-\rho(\delta u)_{x}-\delta u \rho_{x}-u(\delta \rho)_{x} .
$$

Using equations (5.2) we obtain a "non-conservation" form of the L-W scheme, as follows

$$
w^{n+1}=w^{n}-\Delta t A w_{\hat{x}}^{n}+\frac{\Delta t^{2}}{2} \delta^{2} w .
$$

The difference equations (5.1) may be regarded as a difference approximation to the parabolic system

$$
\frac{\partial w}{\partial t}+A \frac{\partial w}{\partial x}=\sigma A^{2} \frac{\partial^{2} w}{\partial x^{2}}
$$

where $\sigma=\Delta t$ (we have assumed that $A$ is constant) [7]. This suggests that the $\mathrm{L}-\mathrm{W}$ scheme adds a "viscosity" to the hyperbolic system. However the stability of the scheme depends on how this "viscosity" term is differenced. If we replace the second derivative by

$$
\frac{w_{j+2}^{n}-2 w_{j}^{n}+w_{j-2}^{n}}{4 \Delta x^{2}}
$$

instead of

$$
\frac{w_{j+1}^{n}-2 w_{j}^{n}+w_{j-1}^{n}}{\Delta x^{2}}
$$


we obtain a new difference approximation for the hyperbolic system which has the same order of accuracy as the L-W scheme. However the scheme is unconditionally unstable as a routine stability analysis will show (this scheme is equivalent to that described in Section 6 with $p=2$ ).

These schemes were tested for accuracy by the method described in Section 3 . The solutions were computed for a supersonic rarefaction wave (Mach number 2.0 ahead and 1.75 behind) and then compared with the analytic solution. The results are given in the table below.

Maximum percentage error at 100 time steps

\begin{tabular}{c|c|c|c}
\hline $\begin{array}{c}\text { Number of } \\
\text { points }\end{array}$ & $\begin{array}{c}\text { Strict } \\
\text { cons. form }\end{array}$ & $\begin{array}{c}\text { Quasi- } \\
\text { cons. form }\end{array}$ & $\begin{array}{c}\text { Non-cons. } \\
\text { form }\end{array}$ \\
\cline { 2 - 3 } 10 & $1.9 \%$ & $2.0 \%$ & $1.6 \%$ \\
20 & .71 & .71 & .69 \\
100 & .015 & .014 & .014 \\
\hline
\end{tabular}

These schemes were tested on flows containing a shock by the method described in Section 4. A compression wave was allowed to develop into a shock, the shock speed was measured, and the computed state behind the shock was compared with that obtained from the Hugoniot relations as a check for consistency. This was done for three different cases yielding shocks of varying strength. The results are given in Table III. In Figure 3 a graph of $p\left(t_{300}, x\right)$ is shown for each scheme. The quasi conservation form has less overshoot than the strict conservation form. This was first noticed by Dr. S. Burstein in a calculation involving two space dimensions [9].

6. The Navier-Stokes Equations. In this section we discuss the application of

TABLE III

Consistency check of the Hugoniot relations

\begin{tabular}{|c|c|c|c|c|c|c|}
\hline & \multicolumn{2}{|c|}{$\begin{array}{c}\text { Strict } \\
\text { cons. form }\end{array}$} & \multicolumn{2}{|c|}{$\begin{array}{l}\text { Quasi } \\
\text { cons. form }\end{array}$} & \multicolumn{2}{|c|}{$\begin{array}{l}\text { Non-cons. } \\
\text { form }\end{array}$} \\
\hline & $\begin{array}{c}\text { Comp. } \\
\text { value }\end{array}$ & $\begin{array}{l}\text { from } \\
R-H\end{array}$ & $\begin{array}{l}\text { Comp. } \\
\text { value }\end{array}$ & $\begin{array}{l}\text { from } \\
\mathrm{R}-\mathrm{H}\end{array}$ & $\begin{array}{l}\text { Comp. } \\
\text { value }\end{array}$ & $\begin{array}{l}\text { from } \\
\mathrm{R}-\mathrm{H}\end{array}$ \\
\hline $\begin{array}{l}\text { Shock Mach No. } \\
\text { Pressure ratio } \\
\text { Density ratio } \\
\text { Velocity ratio }\end{array}$ & $\begin{array}{l}1.62 \\
2.92 \\
2.07 \\
\quad .841\end{array}$ & $\begin{array}{l}2 . \overline{90} \\
2.07 \\
.837\end{array}$ & $\begin{array}{l}1.62 \\
2.92 \\
2.07 \\
\quad .841\end{array}$ & $\begin{array}{l}-\overline{90} \\
2.07 \\
.837\end{array}$ & $\begin{array}{l}1.33 \\
2.69 \\
2.17 \\
\quad .905\end{array}$ & $\begin{array}{l}-\overline{1.90} \\
1.57 \\
.483\end{array}$ \\
\hline $\begin{array}{l}\text { Shock Mach No. } \\
\text { Pressure ratio } \\
\text { Density ratio } \\
\text { Velocity ratio }\end{array}$ & $\begin{array}{l}2.11 \\
5.02 \\
2.82 \\
1.36\end{array}$ & $\begin{array}{l}5 \overline{.00} \\
2.82 \\
1.37\end{array}$ & $\begin{array}{l}2.11 \\
5.02 \\
2.82 \\
1.36\end{array}$ & $\begin{array}{l}5 . \overline{00} \\
2.82 \\
1.37\end{array}$ & $\begin{array}{l}1.57 \\
4.32 \\
8.0 \\
1.51\end{array}$ & $\begin{array}{l}-\overline{2.71} \\
1.98 \\
.777\end{array}$ \\
\hline $\begin{array}{l}\text { Shock Mach No. } \\
\text { Pressure ratio } \\
\text { Density ratio } \\
\text { Velocity ratio }\end{array}$ & $\begin{array}{l}3.00 \\
10.4 \\
3.84 \\
3.70\end{array}$ & $\begin{array}{l}10 . \overline{3} \\
3.85 \\
3.72\end{array}$ & $\begin{array}{l}3.00 \\
10.4 \\
3.84 \\
3.70\end{array}$ & $\begin{array}{c}10 . \overline{3} \\
3.85 \\
3.72\end{array}$ & $\begin{array}{l}1.97 \\
5.14 \\
3.22 \\
4.35\end{array}$ & $\begin{array}{l}-\overline{4} .36 \\
2.62 \\
1.22\end{array}$ \\
\hline
\end{tabular}



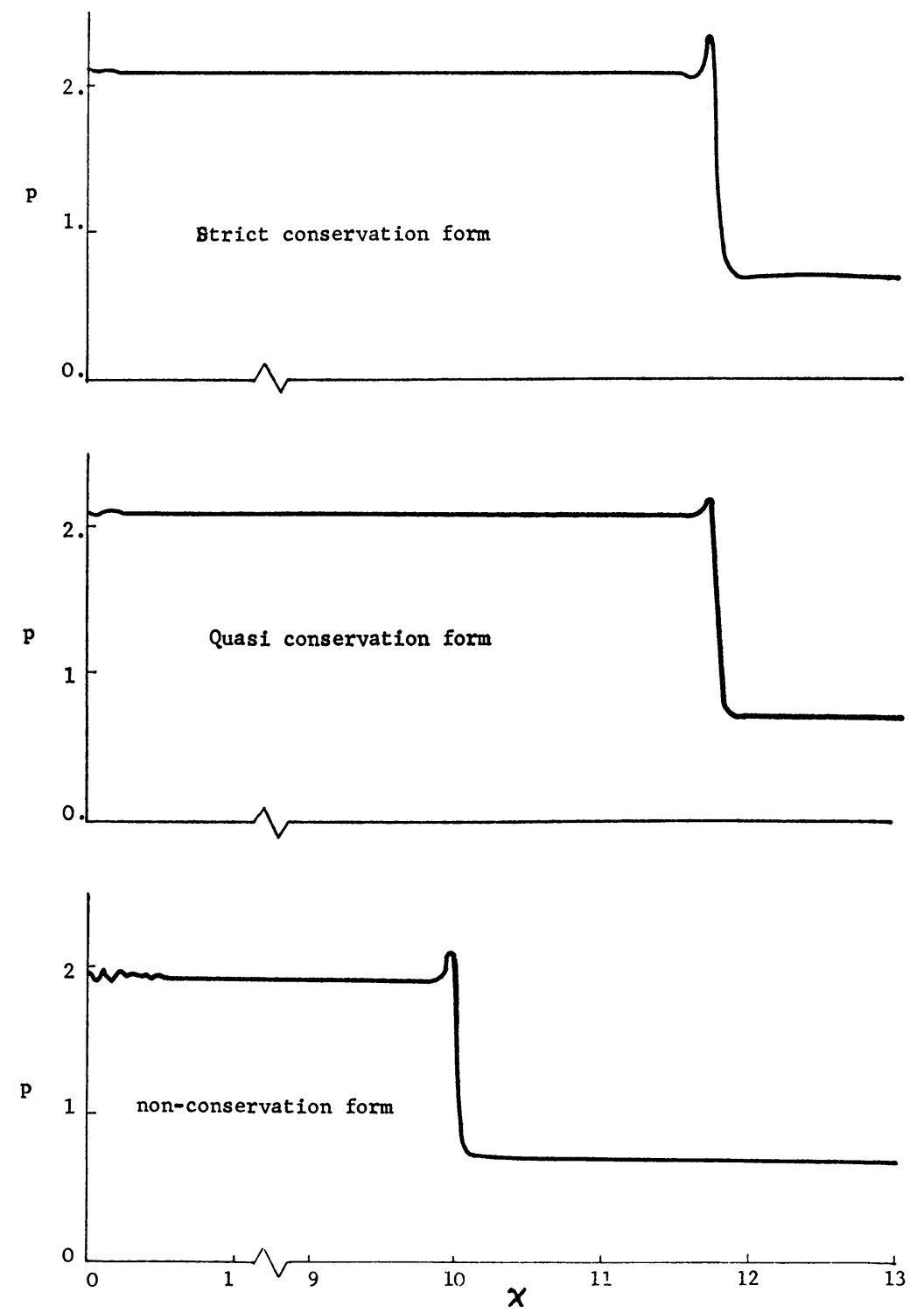
$.05)$.

Fig. 3. Values of pressure computed by Lax-Wendroff scheme after 300 time steps $(\Delta x=$

the Lax-Wendroff method to the Navier-Stokes equations for a viscous fluid. We use the equations below which do not take into account heat conduction. Here $R$ is the Reynolds number.

$$
\begin{aligned}
& \frac{\partial \rho}{\partial t}=-\rho \frac{\partial u}{\partial x}-u \frac{\partial \rho}{\partial x} \\
& \frac{\partial p}{\partial x}=-u \frac{\partial p}{\partial x}-\gamma p \frac{\partial u}{\partial x}+\frac{4(\gamma-1)}{3 R}\left(\frac{\partial u}{\partial x}\right)^{2} \\
& \frac{\partial u}{\partial t}=-u \frac{\partial u}{\partial x}-\frac{1}{\rho} \frac{\partial p}{\partial x}+\frac{4}{3 R \rho} \frac{\partial^{2} u}{\partial x^{2}} .
\end{aligned}
$$


In order to apply the Lax-Wendroff scheme we must have difference approximations for the second derivatives $\partial^{2} \rho / \partial t^{2}, \partial^{2} p / \partial t^{2}$, and $\partial^{2} u / \partial t^{2}$. From the equations of motion we obtain

$$
\frac{\partial^{2} u}{\partial t^{2}}=-u \frac{\partial^{2} u}{\partial x \partial t}-\frac{\partial u}{\partial t} \frac{\partial u}{\partial x}-\frac{1}{\rho} \frac{\partial^{2} p}{\partial x \partial t}+\frac{1}{\rho^{2}} \frac{\partial \rho}{\partial t} \frac{\partial p}{\partial x}+\frac{4}{3 R \rho} \frac{\partial^{3} u}{\partial x^{2} \partial t}-\frac{4}{3 R \rho^{2}} \frac{\partial \rho}{\partial t} \frac{\partial^{2} u}{\partial x^{2}}
$$

and similar equations for $\rho$ and $p$. We let $\delta \rho, \delta p$, and $\delta u$ denote the following difference approximations for the first derivatives $\partial \rho / \partial t, \partial p / \partial t$ and $\partial u / \partial t$ at time $t_{n}$.

$$
\begin{aligned}
& \delta \rho=-\rho^{n} u_{\bar{x}}{ }^{n}-u^{n} \rho_{\bar{x}}{ }^{n} \\
& \delta p=-u^{n}{p_{\bar{x}}}^{n}-\gamma p^{n} u_{\bar{x}}{ }^{n}+\frac{4(\gamma-1)}{3 R} u_{x \bar{x}}^{n} \\
& \delta u=-u^{n} u_{\bar{x}}{ }^{n}-\frac{1}{\rho^{n}}{p_{\bar{x}}}^{n}+\frac{4}{3 R \rho^{n}} u_{x \bar{x}}^{n} .
\end{aligned}
$$

Using the latter in equations (6.2) we obtain difference approximations $\delta^{2} \rho, \delta^{2} p$, and $\delta^{2} u$ for the second derivatives $\partial^{2} \rho / \partial t^{2}, \partial^{2} p / \partial t^{2}$, and $\partial^{2} u / \partial t^{2}$,

$$
\begin{aligned}
\delta^{2} u=-u^{n}(\delta u)_{x}-(\delta u) u_{x}{ }^{n}-\frac{1}{\rho^{n}} & (\delta p)_{x} \\
& \quad+\frac{1}{\left(\rho^{n}\right)^{2}}(\delta \rho) p_{x}^{n}+\frac{4}{3 R \rho^{n}}(\delta u)_{x \bar{x}}-\frac{4}{3 R\left(\rho^{n}\right)^{2}}(\delta \rho) u_{x \bar{x}}^{n}
\end{aligned}
$$

and similar equations for $\rho$ and $p$. The Lax-Wendroff equations are given by

$$
u^{n+1}=u^{n}-\Delta t u^{n} u_{\hat{x}}^{n}-\frac{\Delta t}{\rho} p_{\hat{x}}^{n}+\frac{4}{3 R \rho^{n}} u_{x \bar{x}}^{n}+\frac{(\Delta t)^{2}}{2} \delta^{2} u
$$

and similar equations for $\rho$ and $p$. In order that the truncation error be $O\left(\Delta x^{2}\right)$ the difference approximations used to compute the first derivatives in equation (6.3) must be centered, but the difference approximations used to compute $\delta^{2} \rho$, $\delta^{2} p$, and $\delta^{2} u$ need not be centered. In the calculations the boundary values of $\rho, p$, and $u$, that is, the values at $x_{1}$ and $x_{M}$ are known and the values for $x_{i}, i=2, \cdots$, $M-1$ are computed. Since the second order term in the difference equations requires the values at $x_{i-2}, x_{i-1}, x_{i}, x_{i+1}, x_{i+2}$ and $t^{n}$ to compute the value at $\left(x_{i}, t^{n+1}\right)$, we must modify this term at $i=2$ or $i=M-1$. The modification merely requires that certain forward differences be replaced by backward differences, or conversely.

In order to test this scheme we used the same technique that was described in Section 3 of this report. That is, we let the initial values of $\rho, p$, and $u$ be those in a simple compression wave. Of course the flow is now viscous and the analytic solution for the inviscid case no longer applies. If the Reynolds number is not too large the compression wave will not develop a shock-like structure, but the transition will remain gradual. The use of this problem was suggested by a paper of Ludloff and Filler [8]. They computed the solution for this problem using difference schemes of first order accuracy.

We are unable to compute a stability criterion for the Lax-Wendroff method as applied to the Navier-Stokes equations. We can obtain an empirical stability criterion by computing the solution for a compression wave, for various values of $R, \Delta x$ and $\Delta t$. We compute the solution for 100 time-steps and observe the value of 
TABLE IV

Empirical determination of the stability condition for the Navier-Stokes equations

\begin{tabular}{|c|c|c|c|c|c|}
\hline$R$ & $\Delta x$ & $\begin{array}{c}10^{2} \Delta t \\
(\text { stable })\end{array}$ & $\begin{array}{c}10^{2} \Delta t \\
\text { (unstable) }\end{array}$ & $\begin{array}{c}10^{2} \\
(\Delta x / u+c)\end{array}$ & $10^{2}\left(\frac{1}{2}\right)(3 R \rho / 8) \Delta x^{2}$ \\
\hline $\begin{array}{l}1 \\
1 \\
1\end{array}$ & $\begin{array}{l}0.05 \\
0.025 \\
0.01\end{array}$ & $\begin{array}{l}0.056 \\
0.014 \\
0.0022\end{array}$ & $\begin{array}{l}0.061 \\
0.015 \\
0.0024\end{array}$ & $\begin{array}{l}2.1 \\
1.0 \\
0.42\end{array}$ & $\begin{array}{l}0.047 \\
0.012 \\
0.0019\end{array}$ \\
\hline $\begin{array}{l}10 \\
10 \\
10\end{array}$ & $\begin{array}{l}0.05 \\
0.025 \\
0.01\end{array}$ & $\begin{array}{l}0.56 \\
0.14 \\
0.022\end{array}$ & $\begin{array}{l}0.61 \\
0.15 \\
0.024\end{array}$ & $\begin{array}{l}2.1 \\
1.0 \\
0.42\end{array}$ & $\begin{array}{l}0.47 \\
0.12 \\
0.019\end{array}$ \\
\hline $\begin{array}{l}10^{2} \\
10^{2} \\
10^{2}\end{array}$ & $\begin{array}{l}0.05 \\
0.025 \\
0.01\end{array}$ & $\begin{array}{l}2.0 \\
1.0 \\
0.22\end{array}$ & $\begin{array}{l}2.1 \\
1.1 \\
0.24\end{array}$ & $\begin{array}{l}2.1 \\
1.0 \\
0.42\end{array}$ & $\begin{array}{l}4.7 \\
1.2 \\
0.19\end{array}$ \\
\hline $\begin{array}{l}10^{4} \\
10^{4} \\
10^{4}\end{array}$ & $\begin{array}{l}0.05 \\
0.025 \\
0.01\end{array}$ & $\begin{array}{l}2.1 \\
1.0 \\
0.40\end{array}$ & $\begin{array}{l}2.3 \\
1.1 \\
0.43\end{array}$ & $\begin{array}{l}2.1 \\
1.0 \\
0.42\end{array}$ & $\begin{array}{r}470 \\
120 \\
19\end{array}$ \\
\hline
\end{tabular}

$\Delta t$ for which the computation becomes unstable at fixed values of $\Delta x$ and $R$. We assume the scheme is stable for a given $\Delta t$ if there is no obvious instability after 100 time steps. The results of the computations are shown in Table IV. The largest value of $\Delta t$ for which the calculation is stable is given along with the minimum value of $\Delta t$ for which the calculation is unstable. The simple explicit scheme for the heat equation $\partial u / \partial t=\sigma \partial^{2} u / \partial x^{2}$ has the stability criterion $\Delta t=\left(\frac{1}{2} \sigma\right)(\Delta x)^{2}$. The derivative of second order in the Navier-Stokes equations (2.2) has the coefficient $\frac{4}{3} R \rho$. If the Navier-Stokes equations behave as a parabolic system we might therefore expect the stability criterion $\Delta t=(3 R \rho / 8)(\Delta x)^{2}$. If the behavior is hyperbolic we expect the criterion $\Delta t=(1 /(u+c)) \Delta x$ where the maximum value of $u+c$ at $t=0$ is used. From Table I we see that the behavior is parabolic, that is $\Delta t \sigma$ $(\Delta x)^{2}$, for $R=1$ and $R=10$. It is hyperbolic, $\Delta t \propto \Delta x$ for $R=10^{4}$. As far as these results are concerned we see that the following empirical stability criterion will suffice

$$
\Delta t=\min \left[(\Delta x /(u+c)),\left(\frac{1}{2}\right)(3 R \rho / 8)(\Delta x)^{2}\right] .
$$

We are forced to halve the usual parabolic condition. In Table IV the range of $\Delta t$ in which instability occurs for various $\Delta x$ and $R$ is shown along with the value of $\Delta t$ given by the various stability criteria.

7. An Explicit Difference Scheme. This scheme is based on the Crank-Nicholson method described in Section 2. It is an explicit iterative scheme for the differential equations $\partial w / \partial t+A(w) \partial w / \partial x$ defined as follows. The successive iterates are denoted by $\bar{w}^{(s)}$ and there are exactly $p$ iterations at each time step.

$$
\begin{aligned}
\bar{w}^{(0)} & =w^{n}, \\
\bar{w}^{(s+1)} & =w^{n}-\frac{\Delta t}{2} A\left(\frac{\bar{w}^{(s)}+w^{n}}{2}\right)\left(\bar{w}_{\hat{x}}^{(s)}+w_{\hat{x}}{ }^{n}\right), \\
\bar{w}^{n+1} & =\bar{w}^{(p)},
\end{aligned}
$$


Here we have suppressed the spatial index $j$ in $w_{j}{ }^{n}$, as usual. We may regard this as an iterative method for solving the Crank-Nicholson equations.

We let $w^{+}$be the solution of the Crank-Nicholson equations (assume the matrix $A(w)$ is constant and the boundary conditions are periodic), that is

$$
w^{+}=w^{n}+\frac{\Delta t}{2} A\left(w_{\hat{x}}^{+}+w_{\hat{x}}^{n}\right)
$$

We will show that $\lim _{s \rightarrow \infty} \bar{w}^{(s)}=w^{+}$if $\Delta t<2 \Delta x / \sigma(A)$ where $\sigma(A)$ is the spectral radius of $A$. Define $e_{j}^{(s)}$ by $e_{j}^{(s)}=\bar{w}_{j}^{(s)}-w_{j}{ }^{+}$. Then $e_{j}^{(s)}$ satisfies the equations

$$
e_{j}^{(s+1)}=\frac{\Delta t}{2} A e_{j \hat{x}}^{(s)} .
$$

We let $e^{(s)}$ denote the vector $\left(e_{1}^{(s)}, \cdots, e_{M}{ }^{(s)}\right)$. The periodic boundary conditions yield $e_{1}^{(s)}=e_{M+1}^{(s)}$. We let $Q$ denote the operator defined by $e^{(s)}=a e^{(s-1)}$. If $v$ is an eigenvector of $A$ with eigenvalue $a$ and $k$ is an integer $0 \leqq k \leqq M-1$, then it is easy to see that the vector $e_{j}=v \exp \left(i 2 \pi k j \Delta x /\left(x_{M+1}-x_{1}\right)\right)$ is an eigenvector of a with eigenvalue $i \Delta t \sin (2 \pi k / M) a / 2 \Delta x$. If we take the set of all such $v$ and $k$ then we have a complete set of eigenvectors of $a$. Therefore $\lim _{s \rightarrow \infty} e^{(s)}=0$ and $\lim _{s \rightarrow \infty} \bar{w}^{(s)}=w^{+}$if $\Delta t<\frac{2 \Delta x}{|a|}$ for all eigenvalues of $A$.

We will now analyze the stability of this scheme. We again define the operator a by $a w=\frac{\Delta t}{2} A w_{x}$ where $w$ is a vector whose components are the vectors $\left(w_{1}\right.$, $\left.\cdots, w_{M}\right)$. The difference scheme can be written $\bar{w}^{(s+1)}=w^{n}+a \bar{w}^{(s)}+a w^{n}$. We can show by induction that

$$
w^{n+1}=\left[I+2 Q+\cdots+2 Q^{p}\right] w^{n} .
$$

We now perform a stability analysis by the method of von Neumann. Let $w_{j}{ }^{n}$ $=k^{n} e^{i \omega x_{j}}$, then

$$
Q^{s} w^{n}=\left[\frac{\Delta t i \sin \omega \Delta x}{2 \Delta x} A\right]^{s} w^{n}=(i \mu A)^{s} w^{n}
$$

and

$$
k^{n+1}=\left[I+2 i \mu A+\cdots+2(i \mu A)^{p}\right] k^{n}=M k^{n}
$$

where

$$
\mu=\frac{\Delta t \sin \omega \Delta x}{\Delta x}
$$

Therefore the eigenvalues of $M$ are given by

$$
m=1+2 i \mu a+\cdots+2(i \mu a)^{p}
$$

where $a$ is an eigenvalue of $A$. A little algebra will yield the following results.

$$
m=-1+2\left[\frac{1-i^{p+1}(\mu a)^{p+1}}{1-i_{\mu} a}\right] .
$$






Fig. 4. Velocity computed by two methods when $\Delta x=.05, t=1.12$, shock strength $=$ $\left(p_{1}-p_{0}\right) / p_{0}=.41$.

If $p=2 n+1$,

$$
|m|^{2}=1+(-1)^{n} 4(\mu a)^{2 n+2}\left[\frac{1+(-1)^{n}(\mu a)^{2 n+2}}{1+(\mu a)^{2}}\right] .
$$

If $p=2 n$,

$$
|m|^{2}=1+(-1)^{n+1} 4(\mu a)^{2 n+2}\left[\frac{1+(-1)^{n+1}(\mu a)^{2 n}}{1+(\mu a)^{2}}\right] .
$$

It is clear that the method will be stable only if $\mu|a| \leqq 1$ or $\Delta t<2 \Delta x / a$. Note that this is the condition for $\lim _{s \rightarrow \infty} \bar{w}^{(s)}=w^{+}$above. These relations imply that the scheme will be stable if $p=3,4,7,8, \cdots$ and unstable if $p=1,2,5,6, \cdots$ provided $\Delta t<2 \Delta x / \sigma(A)$. This result has been verified by numerical experiments with this difference scheme. This scheme was used to compute the solution for the same supersonic rarefaction wave that was used to produce Table I in Section 3. For these calculations the value $p=3$ was used. The results are given in the table below. This scheme was also used on a compression wave which eventually developed into a shock. The result is shown in Figure 4. The results for the Lax-Wendroff scheme (in quasi-conservation form) are shown for comparison.

Percentage error after 100 time steps

\begin{tabular}{c|c}
\hline No. of points & Error \\
\hline 10 & 4.2 \\
20 & 1.6 \\
100 & .052 \\
200 & .0081 \\
\hline $\begin{array}{c}\text { Computation time in milliseconds } \\
\text { per mesh point }\end{array}$ & 4.7 \\
\hline
\end{tabular}

New York University

New York 3, New York 
1. R. Courant \& K. O. Friedrichs, Supersonic Flow and Shock Waves, Interscience Publishers, New York, 1948, p. 95.

2. G. E. ForsYTHE \& W. R. WAsow, Finite Difference Methods for Partial Equations, John Wiley and Sons, New York, 1960, p. 237.

3. P. D. LAX \& B. WENDROFP, Difference Schemes with High Order of Accuracy for Solving Hyperbolic Systems, Report NYO-9759, A.E.C. Computing and Applied Math. Center, New York University, 1962.

4. R. D. RIChTMYer, Difference Methods for Initial Value Problems, Interscience Publishers, New York, 1957.

5. 'S. SCHECHTER, "Quasi-tridiagonal matrices and type-insensitive difference equations," Quart. Applied Math., v. 18, 1960, p. 285-295.

6. P. D. LAX \& B. WENDROFF, "Systems of conservation laws," Comm. Pure Appl. Math., v. 13,1960, p. $217-237$.

7. P. D. LAX, "Weak solutions of nonlinear hyperbolic equations and their numerical solution," Comm. Pure Appl. Math. 7, 1954, p. 159.

8. L. FILLER \& H. LUDLOFF, "Stability analysis and integration of the viscous equations of motion," Math. Comp., v. 15, 1961, p. 261-274.

9. S. BuRstEIN, to appear as NYO Report, A. E. C. Computing and Applied Math. Center, New York University.

10. J. Douglas \& B. F. JONEs, "On predictor-corrector methods for non-linear parabolic differential equations," $S I A M$ Jour., v. 11, 1963, p. 195-204. 